\title{
The large scale dust lanes of the Galactic bar
}

\author{
D. J. Marshall ${ }^{1,3}$, R. Fux ${ }^{2, \star}$, A. C. Robin ${ }^{1}$, and C. Reylé ${ }^{1}$ \\ 1 CNRS-UMR 6213, Institut Utinam, Observatoire de Besançon, BP 1615, 25010 Besançon Cedex, France \\ 2 Observatoire de Genève, Université de Genève, 51 Ch. des Maillettes, 1290 Sauverny, Switzerland \\ 3 Département de Physique et Centre Observatoire du Mont Mégantic, Université Laval, Québec, QC, G1K 7P4, Canada \\ e-mail: douglas.marshall.1@ulaval.ca
}

Received 31 October 2007 / Accepted 14 November 2007

\section{ABSTRACT}

\begin{abstract}
Context. Trails of dust inside galactic bars are easily observable in external galaxies. However, information on the dust lanes of the Milky Way bar is harder to obtain due to our position within the Galactic disc.

Aims. By comparing the distribution of dust and gas in the central regions of the Galaxy, we aim to obtain new insights into the properties of the offset dust lanes leading the bar's major axis in the Milky Way.

Methods. On the one hand, the molecular emission of the dust lanes is extracted from the observed CO $l-b-V$ distribution according to the interpretation of a dynamical model. On the other hand, a three dimensional extinction map of the Galactic central region constructed from near-infrared observations is used as a tracer of the dust itself and clearly reveals dust lanes in its face-on projection. Comparison of the position of both independent detections of the dust lanes is performed in the $(l, b)$ plane.

Results. These two completely independent methods are used to provide a coherent picture of the dust lanes in the Milky Way bar. In both the gas and dust distributions, the dust lanes are found to be out of the Galactic plane, appearing at negative latitudes for $l>0^{\circ}$ and at positive latitudes for $l<0^{\circ}$. However, even though there is substantial overlap between the two components, they are offset from one another with the dust appearing to lie closer to the $b=0^{\circ}$ plane.

Conclusions. Two scenarios are proposed to explain the observed offset. The first involves grain destruction by the bar shock and reformation downstream. Due to the decrease in velocity caused by the shock, this occurs at lower $z$. The second assumes that the gas and dust remain on a common tilted plane, but that the molecular gas decouples from the Milky Way's magnetic field, itself strong enough to resist the shear of the bar's shock. The diffuse gas and dust remain coupled to the field and are carried further downstream. This second scenario has recently been suggested in order to explain observations of the barred galaxy NGC 1097.
\end{abstract}

Key words. Galaxy: structure - Galaxy: center - ISM: kinematics and dynamics - ISM: dust, extinction Galaxy: kinematics and dynamics - infrared: ISM

\section{Introduction}

Barred galaxies are ubiquitous in the Universe. Many of these are observed to have dust lanes - trails of gas and dust, seen in extinction, leading the bar's major axis. Evidence for the bar nature of the Milky Way's bulge has been around for over 40 years (de Vaucouleurs 1964). However, our position in our Galaxy does not give us a clear view of the inner regions. Photometric studies are hampered by the high source confusion and severe interstellar extinction whereas gas kinematic studies are rendered difficult due to line of sight crowding of the gas emission lines.

Nevertheless, many studies have obtained information on the Milky Way's dust lanes using a variety of techniques. Calbet et al. (1996) detected a longitude assymetry at $10^{\circ}<|l|<20^{\circ}$ in the DIRBE surface brightness maps which they attributed to the dust lanes. Babusiaux \& Gilmore (2005) used red clump stars to determine the distance as a function of longitude to the bar and using their observations concluded that there is no near-infrared extinction due to the dust lanes in our Galaxy. Other studies have based their interpretation of the observations on models in order to study the chemistry in the Galactic centre (GC) and dust lanes (Rodriguez-Fernandez et al. 2006; Nagai et al. 2007) as well as the physical processes underway in the dust lane shocks (Liszt 2006). However, the results may depend on the assumed

\footnotetext{
* Free-time astronomer.
}

geometry for the dust lanes as no coherent picture currently exists for them.

In this letter, we combine the results from gas dynamics and stellar reddening studies in order to provide a description of the dust lanes leading the bar's major axis. Fux (1999) interpreted the $\mathrm{HI}$ and $\mathrm{CO}$ longitude-velocity diagrams of the Galactic disc using self-consistent $N$-body and smooth particle hydrodynamics simulations of the stellar and gaseous components. Later, Marshall et al. (2006) used a galactic stellar population synthesis model to extract the three dimensional extinction information from near infrared stellar observations. Both studies were able to identify the Milky Way's dust lanes. In the following, we present and analyse the dusty and gassy sky distributions of the dust lanes resulting from these studies.

\section{Dust lanes from 3D extinction maps}

Marshall et al. (2006) created a three dimensional map of the inner Galaxy using the Two Micron All Sky Survey (2MASS) point source catalogue (PSC) (Skrutskie et al. 2006) and the Stellar Population Synthesis Model, developed in Besançon (Robin et al. 2003, which we will simply call "Galactic model"). For a full description of the 3D extinction method, please see Marshall et al. (2006).

Contrary to Marshall et al. (2006), the method used in this study does not have a fixed angular resolution but one that varies 


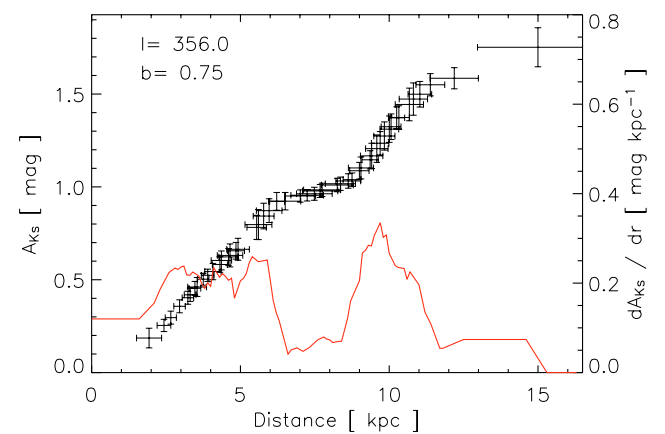

Fig. 1. Application of the extinction method to the line of sight $l=356^{\circ}$, $b=0.75^{\circ}$. The crosses represent the total extinction as a function of distance (left hand scale) and the solid red line is the corresponding differential extinction along the same line of sight (right hand scale). The differential extinction is assumed proportional to the dust density.

as a function of the stellar density. The extinction information for the GC region presented here has an angular resolution that varies from $3.75^{\prime}$ (high density) to $15^{\prime}$ (low density).

The Galactic model used here differs slightly from that described in Robin et al. (2003), as a result of more recent adjustments using 2MASS data. Here, the stellar density of the bulge is modelled using the $\operatorname{sech}^{2}$ function from Freudenreich (1998). It is boxy and prolate, with axis ratios 1:0.3:0.25, and it is assumed to form an angle of $20^{\circ}$ with the Sun - GC direction, in line with recent estimates (Gerhard 2002).

The three dimensional extinction distribution for an example line of sight is shown in Fig. 1. Each cross represents an estimation of the total extinction at the given distance. In order to visualise the three dimensional dust distribution we will present a map of the differential extinction, which is the derivative of the extinction with respect to distance. This will be proportional to the density of the big dust grains $(15 \mathrm{~nm}<$ radius $<110 \mathrm{~nm})$ as they dominate the extinction at $2 \mu \mathrm{m}$ (Désert et al. 1990). The differential extinction for the example line of sight is shown as the solid red line in Fig. 1. Under $\sim 2 \mathrm{kpc}$ and at very large distances, where the distance-extinction points are spaced far apart, it is difficult to identify any underlying structure. However, the technique is well suited to studies of the Galactic bar: the extinction due to the dust lanes for this line of sight can be seen at $r \sim 9.5 \mathrm{kpc}$.

The distribution of dust in the Galactic plane, towards the GC, is displayed in Fig. 2. The elongated structure seen in extinction, running along the stellar bar (shown by the solid line in Fig. 2) but with a slightly different angle, is due to the dust lanes. They can be seen to precede the bar in its clockwise motion (as seen from the NGP), in agreement with observations of dust lanes in external galaxies. The near-side dust lane becomes intertwined with the molecular ring near $x=6 \mathrm{kpc}, y=1 \mathrm{kpc}$ and the far-side dust lane reaches out to distances where the extinction map becomes unreliable or non-existent.

\section{Dust lanes from molecular gas kinematics}

Figure 3 a shows the observed $l-V$ distribution of ${ }^{12} \mathrm{CO}$ in the Galactic bar region. The black contours enclose the two features which have been identified by Fux (1999) as the gaseous signature of the dust lanes along the Galactic bar. The $l>0^{\circ}$ feature, also called "Connecting arm", is the trace of the dust lane in the near-side part of the bar, and the $l<0^{\circ}$ feature is the trace of the far-side dust lane.

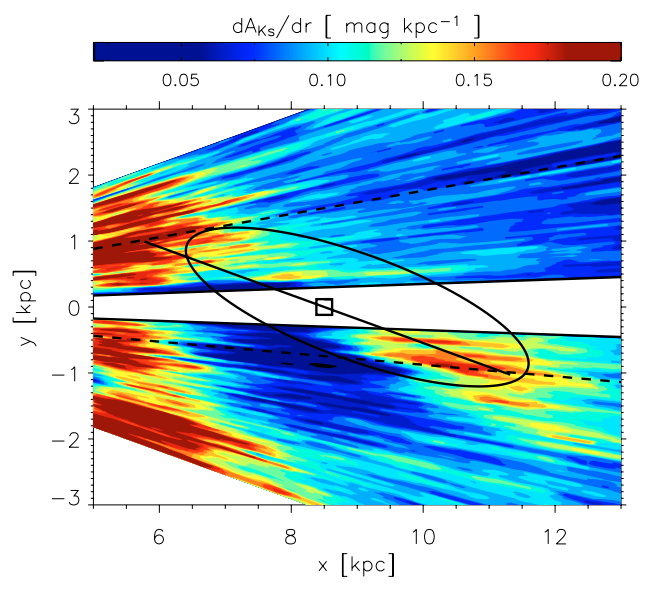

Fig. 2. The average density of absorbing matter in the Galactic bar region for $|z|<300 \mathrm{pc}$, as seen from the NGP. The quantity plotted is the differential extinction (in units of magnitudes per kpc in the $K_{\mathrm{s}}$ band). The GC is indicated by the square symbol, the solid line shows the orientation of the modelled stellar bar, and the ellipse shows the region selected, by inspection, to contain the extinction arising from the dust lanes. The Sun is at $(0,0)$, outside the figure. The dashed lines indicate galactic longitudes $l=-5^{\circ}$ and $l=10^{\circ}$ which approximately delimit the ends of the dust lanes as seen in $\mathrm{CO}$. The white band indicates where the extinction estimates do not fully sample the bar due to the low completeness (high source confusion) of the 2MASS PSC.

Because of the distance indetermination inherent to $l-b-V$ observations, one cannot entirely isolate the emission of these dust lanes from foreground and background emission. The following describes our approach to select most of the dust lanes $\mathrm{CO}$ emission while excluding most of the unrelated emission. The first of the adopted selection criteria restricts the emission to the $l-V$ regions highlighted in Fig. 3a. This criterion avoids in particular the emission of the $|l| \lesssim 1.5^{\circ}$ nuclear ring/disc and of Bania's Clump 1 (Bania 1977) at $(l, V) \approx\left(-5^{\circ}, 100 \mathrm{~km} \mathrm{~s}^{-1}\right)$. It also avoids the emission of the velocity elongated features near $l=3^{\circ}$ (Bania's Clump 2) and $l=5.5^{\circ}$, which may arise from molecular clouds being absorbed by the near-side dust lane (Fux 1999; Liszt 2006; Rodriguez-Fernandez et al. 2006).

The second criterion concerns the gas so far selected in the near-side lane $l-V$ region. The $l-b$ distribution of this gas (Fig. 3b) reveals independent emission within $3.125^{\circ} \leq l \leq$ $10.5^{\circ}$ and $-0.125^{\circ} \leq b \leq 0.625^{\circ}$ (black box in the figure) that forms two distinct $l-V$ features (Fig. 3c). The nearly constant velocity orientation of these features contrasts with the global $l-V$ inclination of the near-side lane and thus suggests that they do not belong to this lane. The gas within the box in Fig. 3b is therefore discarded. Finally, the third selection criterion concerns the gas selected in the far-side lane $l-V$ region. The $b-V$ distribution of this gas (Fig. 3d) separates fairly well the emission of the dust lane from the bright emission of external spiral arms, near $V=0 \mathrm{~km} \mathrm{~s}^{-1}$ and centred at $b \approx 0^{\circ}$, and of the $3-\mathrm{kpc}$ arm, at $(b, V) \approx\left(0^{\circ},-75 \mathrm{~km} \mathrm{~s}^{-1}\right)$. Hence we also exclude all emission between the two lines drawn in Fig. 3d. It should be noted that the remaining emission at $l \gtrsim 8.5^{\circ}$ has characteristics similar to those of the features seen in Fig. $3 \mathrm{c}$ and thus may also not originate from the dust lanes.

\section{Results and discussion}

The sky distribution of the extinction due to the dust lanes is displayed on the top of Fig. 4. The quantity plotted is the integrated extinction owing to all the matter inside the ellipse of Fig. 2. 


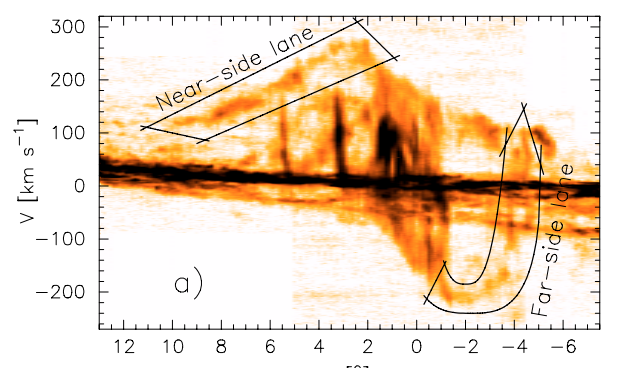

$\left[{ }^{\circ}\right]$

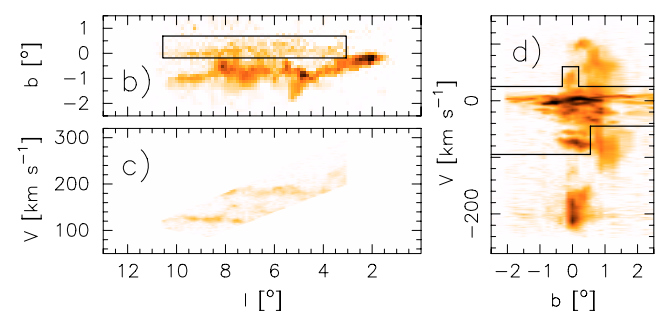

Fig. 3. Selection procedure of the ${ }^{12} \mathrm{CO}$ emission associated with the Galactic bar dust lanes, based on the Dame et al. (2001) $l-b-V$ data cube. a) Longitude-velocity diagram of the $\mathrm{CO}$ gas within $|b| \leq$ $2.5^{\circ}$, with the black contours indicating the near-side (connecting arm) and far-side lane regions set by our first selection criterion. b) Longitude-latitude diagram of the gas within the near-side lane region. c) Longitude-velocity diagram of the gas enclosed by the black box in the former $l-b$ plot; this contribution is rejected according to our second selection criterion. d) Longitude-latitude diagram of the gas in the far-side lane $l-V$ region; our last criterion excludes the emission between the two displayed lines.

We choose sky projections to compare the extinction of the dust lanes with their $\mathrm{CO}$ emission to avoid any modelling required to deproject the raw $\mathrm{CO}$ data. In the bottom of Fig. 4 is the $l-b$ distribution of the gas as selected in Sect. 3. The tilt of the dust lanes relative to the Galactic plane is obvious in both the gas and the dust components, with the negative longitude (far-side) part sitting above the plane and the positive longitude (near-side) part below the plane for both components. Also, both components are well contained within $|l|<10^{\circ}$, showing that we are tracing a different structure to Calbet et al. (1996).

The mean plane of the dust/gas as a function of galactic longitude is given by $\bar{b}(l)=\sum_{b} b \cdot X(l, b) / \sum_{b} X(l, b)$ where $X(l, b)$ is either the extinction due to dust or the intensity of the $\mathrm{CO}$ emission emanating from the dust lanes. The calculation of the mean plane for the dust uses lines of sight where $A_{K_{\mathrm{s}}} \geq 0.15$ (indicated by the solid contour in the top image of Fig. 4), in order to avoid low density lines of sight unrelated to the dust lanes. The mean planes of the dust and the gas, calculated this way, are shown in Fig. 5. Both the gas and the dust follow the same trend, however the dust is seen to be displaced from the gas.

The magnitude of the apparent displacement is larger at positive longitudes than at negative longitudes. The removal from the $\mathrm{CO}$ observations of the zone highlighted in Fig. $3 \mathrm{~b}$ may bias the mean plane to more negative latitudes but, as the bulk of the dust lane $\mathrm{CO}$ emission arises from outside of this zone, the effect would be small. Likewise, the contribution of the dust component outside the dust lanes has been minimised using the high extinction lines of sight chosen using the ellipse of Sect. 2. In fact, the difference in apparent displacement is largely an effect of perspective, as the near side of the bar lies at positive longitudes.

These results suggest that, even though the large scale morphology of the dust lanes is similar for the two components
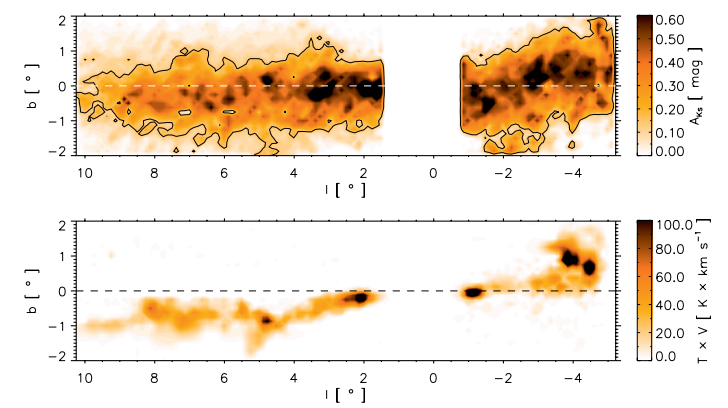

Fig. 4. Longitude-latitude distributions of the near-infrared absorbing dust lanes detected in the Galactic bar by Marshall et al. (2006) (top), and of the corresponding ${ }^{12} \mathrm{CO}$ emission derived from the Dame et al. (2001) data assuming the $l-V$ feature interpretation given by Fux (1999) (bottom). Both images are filled contour plots with $7.5^{\prime}$ resolution. The Galactic $b=0^{\circ}$ plane is indicated by the dashed line. The $\mathrm{CO}$ emission from the central molecular disc has not been included (see Sect. 3), and the dust map excludes the area masked out in Fig. 2. The contour represents $A_{K_{\mathrm{s}}}=0.15 \mathrm{mag}$. Both the gas and dust are seen to be tilted with respect to the Galactic plane, with the negative longitude (far) side sitting above the plane and the positive longitude (near) side sitting below.

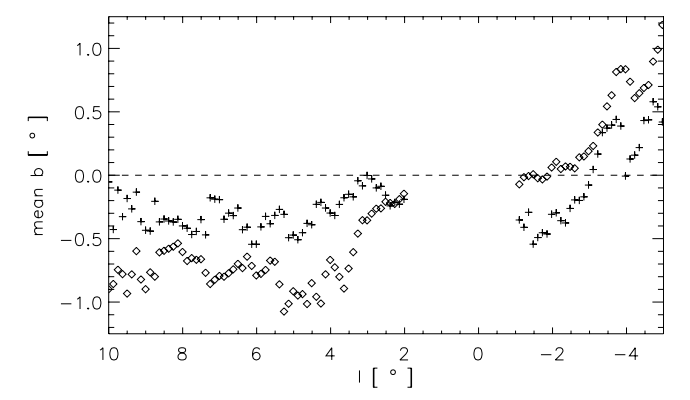

Fig. 5. Position of the mean plane of dust (crosses) and molecular gas (diamonds) along the dust lanes in the Galactic bar. The $b=0^{\circ}$ plane is shown by the dashed line. The same large scale tilt is observed for both the gas and dust, however the dust appears closer to the $b=0^{\circ}$ plane.

studied here, there is an additional effect which causes their $l, b$ projections to be offset from one another. This could be due to an offset in $z$, a separation in the $x, y$ coordinates of a tilted plane or a combination of the two.

Offsets have been detected between dust and gas in external galaxies seen face on, where azimuthal offsets are easier to discern than offsets in $z$. For example, in M 83 the dust extinction and $\mathrm{CO}$ emission in the eastern spiral arm are offset by as much as 700 pc (Lord \& Kenney 1991; Rand et al. 1999). Three explanations are offered to explain the offset: 1) the $\mathrm{CO}$ is heated by UV radiation from young stars; 2) the $\mathrm{CO}$ is heated by lowenergy cosmic rays; 3 ) the components react differently to the spiral density wave. In the case of the Milky Way bar, the first option is unlikely as star formation along the bar is likely hampered by the high shear there (Rodriguez-Fernandez et al. 2006), and stars formed at the ends of the bar are not expected to migrate along it (Cole \& Weinberg 2002). Cosmic ray heating may be responsible for increased gas temperatures in the centre of the Galaxy (Yusef-Zadeh et al. 2007) but these temperatures can also be explained by shock waves (Lis et al. 2001). In the third case, the diffuse gas is trapped in a thin dust lane at the shock front and the molecular clouds form a broad ridge on the spiral arm. However this does not seem to correspond to our observations, where it is the dust that is seen to be more broadly distributed than the molecular gas (Fig. 4). 
Recently, Beck et al. (2005) observed the barred galaxy NGC 1097 in total and polarised intensity at $\lambda=3.5 \mathrm{~cm}$ and $\lambda=6.2 \mathrm{~cm}$. They found that around the bar the total intensity increases along the shearing shock, however the increase of the polarised emission occurs further downstream and is aligned with the bar's dust lanes. They suggest that the large scale magnetic field is only weakly affected by the shock and that the molecular clouds become decoupled from the magnetic field and are thus swept up by the bar's shearing shock. The dust, coupled to the magnetic field, thus finds its way $\sim 400 \mathrm{pc}$ downstream from the CO. Furthermore, they suggest that this may occur in most disc galaxies where large scale shocks trigger the formation or collapse of gas clouds. Our observations are consistent with this scenario, assuming that the gas and dust of the dust lanes orbit on a common and tilted plane. However, Beck et al. (2005) were not able to obtain direct observations of the molecular gas - dust separation in this galaxy. In order to detect an offset of a few hundred parsecs between $\mathrm{CO}$ and dust in an external galaxy would require high resolution and high sensitivity $\mathrm{CO}$ observations of a nearby barred galaxy with prominent dust lanes.

Instead of considering an offset on a common plane, it is possible that the observed offset is in effect a separation in $z$. As the gas and dust hit the shearing shock, it is possible that a fraction of this gas and dust fall towards the Galactic plane. Two possible mechanisms could be considered. Firstly, Gómez \& Cox (2004) show using 3D MHD simulations that gas hitting a spiral arm shock can be deflected over the arm. Although there has been no study of this kind done on the bar shocks, there is also no evidence indicating that the gas and dust should be confined to the same plane. Secondly, the molecular gas and dust enter the shock front and merge with the dust lane at a given height above the Galactic plane. Due to the gravitational potential, the postshock material eventually falls onto the Galactic plane creating a vertical distribution of azimuthally comoving gas and dust in the dust lane.

These mechanisms could explain a vertical distribution of gas and dust at the shock fronts but not a separation between them. However, at the shock front the big grains of the dust (responsible for the NIR extinction) are shattered (Jones et al. 1994), creating many smaller grains (responsible for UV extinction). As the gas moves away from the shock front, the increased density speeds up the rate of coagulation between grains and, in particular, small grains on large ones. If some of the gas and dust are heading towards the Galactic plane, the lack of $\mathrm{CO}$ at low $z$ could be explained by two processes: 1) accretion of gaseous $\mathrm{CO}$ on to grains as an ice mantle, which is efficient for $A_{K_{\mathrm{s}}} \gtrsim 0.35$ (Draine 2003); 2) dissociation of CO due to the increase in UV photon flux resulting from the decrease in the small grain abundance. The dust layer would be found at systematically lower $z$ than the CO, as can be seen in Figs. 4 and 5 .

Fux (1999) represented the tilts of the $\mathrm{CO}$ and $\mathrm{HI}$ by a straight line with an angle of $\theta \approx 4.5^{\circ}$ at their azimuth of $25^{\circ}$, while mentioning that this representation is an oversimplification. The tilt of the dust component is not as easily represented by a straight line (Fig. 5). Its value is highly dependent on the segment of the dust lane which is fitted and the interpretation of its value depends on the scenario used to explain the offset discussed above.

Finally, there are uncertainties in the dust position, namely in its distance along the line of sight. This uncertainty is estimated to be about $500 \mathrm{pc}$ in the bar (Fig. 1). Moreover the computation of the 3D extinction map made use of the Galactic model, hence it depends slightly on the parameters of this model (Marshall et al. 2006). This dependence might cause a systematic error of a few $100 \mathrm{pc}$ in the distance of the dust along the line of sight, but not on its $(l, b)$ position. It is expected that this error would affect the positive and negative longitudes similarly, having no influence on the tilt of the dust lanes in the $(l, b)$ plane.

\section{Conclusions}

We have identified the dominant dust lanes of the Galactic bar both by their near-infrared extinction and by their $\mathrm{CO}$ emission. The same large scale behaviour is observed in both components, that is a tilt going from $b<0^{\circ}$ at positive $l$ to $b>0^{\circ}$ at negative $l$. In the $l, b$ plane projections, the two components are seen to be displaced from one another. This offset may be due to dust destruction at the shock front followed by reformation and/or ice mantle accretion downstream and at lower $z$, or the molecular gas becoming decoupled from the magnetic field which resists the shearing shock of the Milky Way's bar and carries diffuse gas and dust further downstream on a tilted plane. Further observations of our Galaxy and external galaxies, together with theoretical work are necessary in order to unambiguously interpret these observations.

Finally, the coincidence between the two independently recovered gas and dust distribution along the bar reinforces the interpretation from Fux (1999) regarding the $l-V$ traces of the dust lanes in the observed HI and CO kinematics, and it adds credibility to the Galactic model and the use of it to determine the three dimensional extinction distribution.

Acknowledgements. The authors would like to thank G. Joncas, A. Jones, V. Guillet and A. Fletcher for helpful discussions. D.J. Marshall was funded for part of this work by the Natural Sciences and Engineering Research Council of Canada through its SRO programme. This publication makes use of data products from the Two Micron All Sky Survey, which is a joint project of the University of Massachusetts and the Infrared Processing and Analysis Center/California Institute of Technology, funded by the National Aeronautics and Space Administration and the National Science Foundation. The CDSClient package was used for the remote querying of the 2MASS dataset.

\section{References}

Babusiaux, C., \& Gilmore, G. 2005, MNRAS, 358, 1309

Bania, T. M. 1977, ApJ, 216, 381

Beck, R., Fletcher, A., Shukurov, A., et al. 2005, A\&A, 444, 739

Calbet, X., Mahoney, T., Hammersley, P. L., Garzon, F., \& Lopez-Corredoira, M. 1996, ApJ, 457, L27

Cole, A. A., \& Weinberg, M. D. 2002, ApJ, 574, L43

Dame, T. M., Hartmann, D., \& Thaddeus, P. 2001, ApJ, 547, 792

de Vaucouleurs, G. 1964, in The Galaxy and the Magellanic Clouds, ed. F. J. Kerr, IAU Symp., 20, 195

Désert, F.-X., Boulanger, F., \& Puget, J. L. 1990, A\&A, 237, 215

Draine, B. T. 2003, ARA\&A, 41, 241

Freudenreich, H. T. 1998, ApJ, 492, 495

Fux, R. 1999, A\&A, 345, 787

Gerhard, O. 2002, in ASP Conf. Ser. 273, ed. G. S. Da Costa, \& H. Jerjen, 73 Gómez, G. C., \& Cox, D. P. 2004, ApJ, 615, 744

Jones, A. P., Tielens, A. G. G. M., Hollenbach, D. J., \& McKee, C. F. 1994, ApJ, 433, 797

Lis, D. C., Serabyn, E., Zylka, R., \& Li, Y. 2001, ApJ, 550, 761

Liszt, H. S. 2006, A\&A, 447, 533

Lord, S. D., \& Kenney, J. D. P. 1991, ApJ, 381, 130

Marshall, D. J., Robin, A. C., Reylé, C., Schultheis, M., \& Picaud, S. 2006, A\&A, 453, 635

Nagai, M., Tanaka, K., Kamegai, K., \& Oka, T. 2007, PASJ, 59, 25

Rand, R. J., Lord, S. D., \& Higdon, J. L. 1999, ApJ, 513, 720

Robin, A. C., Reylé, C., Derrière, S., \& Picaud, S. 2003, A\&A, 409, 523

Rodriguez-Fernandez, N. J., Combes, F., Martin-Pintado, J., Wilson, T. L., \& Apponi, A. 2006, A\&A, 455, 963

Skrutskie, M. F., Cutri, R. M., Stiening, R., et al. 2006, AJ, 131, 1163

Yusef-Zadeh, F., Wardle, M., \& Roy, S. 2007, ApJ, 665, L123 